\title{
Image-Guided Ablation of Malignant Liver Tumors: Recommendations for Clinical Validation of Novel Thermal and Non-Thermal Technologies - A Western Perspective
}

\author{
Riccardo Lencionia, Thierry de Baere ${ }^{\mathrm{b}} \quad$ Robert C. Martin ${ }^{\mathrm{c}}$ \\ Charles W. Nutting ${ }^{d}$ Govindarajan Narayanane
}

${ }^{a}$ Division of Diagnostic Imaging and Intervention, Pisa University School of Medicine, Pisa, Italy ${ }^{b}$ Department of Interventional Radiology, Institut Gustav-Roussy, Villejuif Cedex, France, 'Division of Surgical Oncology, University of Louisville, Louisville, Ky., dSky Ridge Medical Center, Lone Tree, Colo., eDivision of Vascular Interventional Radiology, University of Miami Miller School of Medicine, Miami, Fla., USA

\section{Key Words}

Cryoablation · Hepatocellular carcinoma · Irreversible electroporation · Microwave ablation - Radiofrequency ablation

\begin{abstract}
Background: Image-guided ablation is used to treat patients with unresectable malignant hepatic tumors that are limited in number and size, especially hepatocellular carcinoma (HCC) and colorectal hepatic metastases. While radiofrequency ablation (RFA) has been the most popular technique, several alternate options for focal tissue destruction have recently attracted attention. These technologies appear to be able to overcome some specific limitations of RFA. Currently, there is no accepted algorithm for the use of the different techniques for image-guided ablation. Summary: A panel of physicians practicing in North America or Europe met to develop a set of recommendations aimed at providing directions for clinical validation of energy-based, thermal and non-thermal image-guided ablation technologies in the treatment of malignant liver tumors. The recommendations were developed through a critical appraisal of potential advantages and disadvantages of each ablation technology, based on experimental findings and available data, as well as on critical considerations for
\end{abstract}


their clinical validation in hepatic tumor treatment from a Western perspective. Key Messages: Significant variability appears to exist among the different equipment and devices within each type of technology. A comprehensive understanding of the data and a critical appraisal of the efficacy and safety profile of each ablation system is required. Clinical practice guidelines should include specific information of the recommended techniques and protocols instead of a generic indication of the technology.

Copyright $@ 2015$ S. Karger AG, Basel

\section{Introduction}

In recent years, the use of image-guided ablation to treat primary and secondary liver tumors has increased worldwide. Hepatocellular carcinoma (HCC) is currently the second leading cause of cancer-related death and its future incidence and mortality rates are projected to further increase over the forthcoming decades [1]. The development of hepatic metastases is the main cause of death in patients with colorectal carcinoma, a malignancy affecting more than 1.3 million people worldwide annually [1]. Unfortunately, despite the progress of modern surgical techniques, resection of primary and secondary liver tumors is only possible in a minority of patients because of the associated extrahepatic disease, extent and location of the lesions in the liver, impaired liver function, or concurrent medical conditions [2,3]. Image-guided ablation is currently used to treat nonsurgical patients with early-stage HCC or liver-only or liver-dominant metastatic disease that is limited in number and size $[4,5]$.

Position statements and quality improvement guidelines on image-guided ablation of liver tumors focused on the use of monopolar radiofrequency ablation (RFA), since this technology is supported by a large amount of data and robust clinical evidence [6, 7]. However, several alternate options for focal tissue destruction have recently attracted attention, since they appear to be able to overcome some specific limitations of RFA [8-10]. These novel energy-based ablation technologies destroy a tumor either through thermal (hot or cold) or non-thermal mechanisms.

Currently, there is no accepted algorithm for the use of the different ablative techniques in the treatment of liver tumors. Further studies are needed to establish whether novel technologies will expand the clinical role of image-guided ablation and improve long-term patient outcomes with respect to RFA. With this in mind, a panel of expert physicians met to develop a set of recommendations aimed at providing directions for the clinical validation of energy-based, thermal and non-thermal image-guided ablation technologies in the treatment of malignant liver tumors.

\section{The Panel}

The group of physicians, all having considerable experience in the field, included four interventional radiologists and one surgical oncologist, practicing in North America $(n=3)$ or Europe $(\mathrm{n}=2)$. The recommendations were collaboratively generated during a person-toperson meeting. 


\section{General Considerations}

The panel underscores that accurate patient selection is key to the success of image-guided ablation treatment with any technology and in any tumor histology. Indications and contraindications to image-guided ablation should follow available clinical practice guidelines. Eligibility for image-guided ablation treatment should ultimately be established in each individual patient by a multidisciplinary team based on comprehensive clinical, imaging, and laboratory assessment.

\section{Choice of Approach}

Image-guided ablation can be performed with a percutaneous, laparoscopic, or an open surgical approach, although clear advantages of one over the other may apply in specific clinical circumstances. The choice of the most appropriate approach in each individual patient should be at the physician's discretion, since it depends on the combination of several factors, including, but not limited to, the general patient condition, treatment plan, tumor histology, and number, size, and location of the target lesions.

\section{Imaging Guidance and Monitoring}

Tumor ablation can be guided by ultrasound, computed tomography (CT), or magnetic resonance imaging (MRI). The choice of the imaging guidance should be at the physician's discretion, since it depends on the combination of several factors, including, but not limited to tumor visibility, operator preference, and local availability of dedicated equipment, such as CT fluoroscopy or open MRI systems. Preprocedural assessment scans should be less than two weeks old. Detailed confirmatory imaging at the time of treatment will facilitate complete ablation. Checking ablation results at the time of treatment by the use of contrast enhanced studies, will increase the chances of complete ablation with adequate margins in all dimensions.

\section{Ablation Strategy}

The ablation of appropriate margins beyond the visible borders of the tumor is necessary to achieve therapeutic results similar to those achieved with surgery. Ideally, a 360-degree, 0.5 to $1.0 \mathrm{~cm}$-thick ablative margin should be produced all around the target tumor. This cuff is aimed at ensuring that the peripheral portion of the lesion as well as any microscopic invasion located in its close proximity have been eradicated $[6,7]$.

It has been shown that obtaining ablative margins thicker than $1 \mathrm{~cm}$ is even better to further reduce the likelihood of local tumor progression in colorectal liver metastases [11]. Margins may be particularly important where tumor definition is poor.

The following considerations deserve attention when planning the ablation strategy: 1. Ablation protocols are usually based on the ablation size charts provided by the manufacturer. However, the actual ablation zone may vary in the clinical setting, depending upon the tissue vascularization, the tissue conductivity, local interactions, and the settings of the system among other factors.

2. Tumors are generally assumed to be spherical. If the difference between the longest axis and the shortest axis of a tumor is $1 \mathrm{~cm}$ or greater, appropriate changes to the ablation strategy may be warranted in view of the ellipsoidal shape of the target.

3. Changes in the actual position of the electrodes with respect to the planned position can potentially lead to incomplete ablation or thermal injury of structures located in the vicinity of the target tumor; appropriate changes to the ablation protocol may be warranted based on the actual position of the electrodes.

4. Imaging aspects that are particularly important when planning the ablation strategy in- 
clude the tumor size and the shape and location within the organ relative to the blood vessels as well as to critical structures that might be at risk for injury; an individualized ablation protocol may be required depending on the location of the tumor and its relationship with blood vessels as well as to critical structures that might be at risk for injury.

\section{Energy-Based Ablation Technologies}

The term "tumor ablation" is defined as the direct application of chemical (i.e., nonenergy) or energy-based (i.e., thermal and non-thermal) therapies to eradicate or substantially destroy focal tumors [12]. The present document is focused on energy-based ablation technologies. The authors acknowledge that chemical ablation by using percutaneous injection of absolute ethanol is an established technique for the treatment of small HCC lesions. However, the superiority of RFA over ethanol ablation in the treatment of early-stage HCC has already been shown by randomized controlled trials and meta-analyses [2, 4]. Energybased ablation includes technologies that destroy a tumor either through thermal (hot or cold) or non-thermal mechanisms.

RFA

RFA has been the most widely assessed technology for local ablation of liver tumors $[6,7]$. The available RFA systems function in the $375-500 \mathrm{KHz}$ range [12]. Most devices currently used are monopolar, in that there is a single active electrode, with current dissipated at one or more return grounding pads. Bipolar devices have two active electrode applicators, usually placed in close proximity to achieve contiguous coagulation between the them, or on a single electrode. The ability of RFA to achieve complete tumor eradication appears to be dependent on the tumor size and location. Histological studies have shown that a tumor diameter greater than $3 \mathrm{~cm}$ and a perivascular location (defined as tumor adjacent to vessels of $3 \mathrm{~mm}$ or more in caliber) result in a substantial drop in the rate of complete tumor ablation [4]. Combined approaches including transarterial chemoembolization and RFA have been used to increase the efficacy of RFA in the treatment of HCC tumors of intermediate or large size [13].

\section{Microwave Ablation (MWA)}

MWA is a promising heat-based thermal ablation modality that has particular applicability in treating hepatic malignancies. Microwaves can generate very high temperatures in a very short time, potentially leading to improved treatment efficiency and larger ablation zones, with less susceptibility to heat-sink effects [8]. Currently available MWA systems function at $915 \mathrm{MHz}$ or $2.45 \mathrm{GHz}$ frequencies [12]. These wavelengths have specific physical properties with regard to tissue permeability and antenna design. Multiple microwave antennas can be powered simultaneously to take advantage of thermal synergy when placed in close proximity to treat a large tumor or widely spaced to ablate several tumors simultaneously. As the available technology continues to improve, MWA is emerging as a valuable alternative to RFA in the treatment of hepatic malignancies $[14,15]$.

\section{Cryoablation}

This term should be exclusively used for all methods of destroying tissue by the application of low temperature freezing, or alternating freezing and thawing [12]. Rapid tissue freezing and thawing produces the greatest cytotoxic effects by disrupting cellular membranes and inducing cell death [12]. Advantages of cryoablation include the ability to moni- 
tor the ice-ball formation during the procedure on imaging and the absence of pain when delivering the treatment [8]. Potential disadvantages include a severe post-treatment systemic inflammatory response syndrome termed cryoshock (typically seen with large-volume liver cryoablation) and the lack of cautery effects and coagulation of injured vessels, which can predispose to bleeding complications [8]. The technique has had relatively limited application in the treatment liver malignancies so far, and efficacy and safety profiles are currently being investigated [16].

\section{Irreversible Electroporation (IRE)}

The term IRE should be used for those technologies and devices that cause cell death through the repeated application of short duration high voltage electrical pulses that create irreversible injuries to cellular membranes [12]. While there may be some hyperthermic ablative changes with higher power applications, the mechanism of cell death with IRE is thought to be predominantly non-thermal [12]. Hence, issues associated with perfusion-mediated tissue cooling are not relevant for this technology. IRE is administered under general anesthesia with administration of a neuromuscular blocking agent to prevent undesirable muscle contraction and by using cardiac gating to synchronize pulse delivery with absolute refractory period to prevent cardiac arrhythmias. Initial clinical experiences have suggested that IRE preserves the structural integrity of bile ducts and vessels and can enable safe treatment of hepatic tumors located in the proximity to vital structures [17-19].

\section{Other Technologies}

Other energy-based ablation technologies include laser ablation and high intensity focused ultrasound. These technologies have been adopted by few centers worldwide for the treatment of malignant liver tumors. Hence, limited data are available concerning their efficacy and safety profiles, and further clinical investigation is warranted.

\section{Recommendations for Clinical Validation}

The recommendations for clinical validation of energy-based, thermal and non-thermal image-guided ablation technologies in the treatment of malignant liver tumors were developed through a critical appraisal of the potential advantages and disadvantages of each thermal and non-thermal ablation technology, based on experimental findings and available data (table 1).

Critical considerations for clinical validation of ablation technologies in hepatic tumor treatment include:

- Monopolar RFA is an established technique for the treatment of tumors that are limited in number ( 3 or less) and size ( $3 \mathrm{~cm}$ or less) and are located $1 \mathrm{~cm}$ or more from critical structures and vessels. Vessels $3 \mathrm{~mm}$ or more in caliber are considered relevant for heat-sink effect. - MWA appears to have the potential to improve the rate of complete ablation achieved with RFA in tumors that are larger than $3 \mathrm{~cm}$ or when multiple; device-specific safety and efficacy data including predictability and reproducibility are warranted.

- MWA seems to have the potential to overcome the limitations of RFA in the treatment of tumors in perivascular locations; device-specific safety and efficacy analyses are warranted

- IRE shows promise for the treatment of small tumors located in the vicinity of critical structures; device-specific safety and efficacy data are warranted

- More data is needed to define the potential of other energy-based ablation technologies in the specific field of liver tumor treatment 
Table 1. Main potential advantages and disadvantages of each energy-based ablative technology in hepatic tumor treatment

\begin{tabular}{|c|c|c|}
\hline Technology & Potential Advantages & Potential Disadvantages \\
\hline RFA & $\begin{array}{l}\text { - High rates of local control in tumors } 3 \mathrm{~cm} \\
\text { or smaller } \\
\text { - Established safety profile } \\
\text { - Known limitations } \\
\text { - Experience in combination treatments } \\
\text { (HCC) } \\
\text { - Widely available }\end{array}$ & $\begin{array}{l}\text { - High rates of incomplete ablation in } \\
\text { tumors larger than } 3 \mathrm{~cm} \\
\text { - Heat sink effect in perivascular tumors } \\
\text { - Potential risk of thermal injury to criti- } \\
\text { cal structures } \\
\text { - Variability in RFA devices }\end{array}$ \\
\hline MWA & $\begin{array}{l}\text { - Potential to treat tumors larger than } 3 \mathrm{~cm} \\
\text { more effectively } \\
\text { - Less impacted by heat sink effect } \\
\text { - Ability to activate multiple probes at the } \\
\text { same time } \\
\text { - No grounding pads required }\end{array}$ & $\begin{array}{l}\text { - Limited efficacy data (predictability } \\
\text { and reproducibility) } \\
\text { - Limited safety data } \\
\text { - Potential risk of thermal injury to criti- } \\
\text { cal structures (and vessels?) } \\
\text { - Variability in MWA devices }\end{array}$ \\
\hline CRYO & $\begin{array}{l}\text { - Ability to activate multiple probes at the } \\
\text { same time } \\
\text { - Ability to image the ice-ball formation }\end{array}$ & $\begin{array}{l}\text { - Insufficient clinical data } \\
\text { - Risk of bleeding } \\
\text { - Risk of cryoshock }\end{array}$ \\
\hline IRE & $\begin{array}{l}\text { - Potential to treat tumors located in the } \\
\text { vicinity of critical structures } \\
\text { - Heat sink effect not relevant }\end{array}$ & $\begin{array}{l}\text { - Insufficient clinical data } \\
\text { - Neuromuscular blockage and cardiac } \\
\text { gating required }\end{array}$ \\
\hline
\end{tabular}

It is important to note that recommendations and directions refer to the general characteristics of each technology; however, significant variability appears to exist among the different equipment and devices. Therefore, detailed information should be provided concerning technique parameters to enable a comprehensive understanding of the data and a critical appraisal of the efficacy and safety profile of each ablation system. Ultimately, as opposed to a generic indication of the technology, specific information of the recommended techniques and protocols should be implemented in clinical practice guidelines, similar to pharmaceutical treatment regimens.

\section{Acknowledgements}

The authors would like to thank the Research Analysis Library for providing a draft based on the minutes of the meeting. Assistance was supported by AngioDynamics Inc.

\section{Disclosure Statement}

The authors declare no conflict of interest.

\section{References}

1 International Agency for Research on Cancer: World Health Organization. GLOBOCAN 2012: Estimated cancer incidence, mortality, and prevalence worldwide in 2012. http://globocan.iarc.fr (accessed January 20, 2015). 
2 Bruix J, Sherman, M American Association for the Study of Liver Diseases: Management of hepatocellular carcinoma: an update. Hepatology 2011;53:1020-1022.

3 Adams RB, Aloia TA, Loyer E, Pawlik TM, Taouli B, Vauthey JN: Selection for hepatic resection of colorectal liver metastases: expert consensus statement. HPB Oxf 2013;15:91-103.

4 Lencioni R, Crocetti L: Loco-regional treatment of hepatocellular carcinoma. Radiology 2012;262:43-58.

5 Mahnken AH, Pereira PL, de Baere T: Interventional oncologic approaches to liver metastases. Radiology 2013;266:407-430.

6 Gervais DA, Goldberg SN, Brown DB, Soulen MC, Millward SF, Rajan DK: Society of Interventional Radiology position statement on percutaneous radiofrequency ablation for the treatment of liver tumors. J Vasc Interv Radiol 2009;20(Suppl):S342-S347.

7 Crocetti L, de Baere T, Lencioni R: Quality improvement guidelines for radiofrequency ablation of liver tumours. Cardiovasc Intervent Radiol 2010;33:11-17.

8 Lubner MG, Brace CL, Ziemlewicz TJ, Hinshaw JL, Lee FT Jr: Microwave ablation of hepatic malignancy. Semin Intervent Radiol 2013;30:56-66.

9 Lu DS, Kee ST, Lee EW: Irreversible electroporation: ready for prime time? Tech Vasc Interv Radiol 2013;16:277-286.

10 Erinjeri JP, Clark TW: Cryoablation: mechanism of action and devices. J Vasc Interv Radiol 2010;21:S187S191.

11 Wang X, Sofocleous CT, Erinjeri JP, et al: Margin size is an independent predictor of local tumor progression after ablation of colon cancer liver metastases. Cardiovasc Intervent Radiol 2013;36:166-175.

12 Ahmed M, Solbiati L, Brace CL, et al: Image-guided tumor ablation: standardization of terminology and reporting criteria - a 10-year update. Radiology 2014;273:241-260.

13 Peng ZW, Zhang YJ, Chen MS, et al: Radiofrequency ablation with or without transcatheter arterial chemoembolization in the treatment of hepatocellular carcinoma: a prospective randomized trial. J Clin Oncol 2013;31:426-432.

14 Martin RC, Scoggins CR, Mcmasters KM: Safety and efficacy of microwave ablation of hepatic tumors: a prospective review of a 5-year experience. Ann Surg Oncol 2010;17:171-178.

15 Groeschl RT, Pilgrim CH, Hanna EM, et al.: Microwave ablation for hepatic malignancies: a multiinstitutional analysis. Ann Surg 2014;259:1195-1200.

16 Dunne RM, Shyn PB, Sung JC, et al: Percutaneous treatment of hepatocellular carcinoma in patients with cirrhosis: a comparison of the safety of cryoablation and radiof requency ablation. Eur J Radiol 2014;83:632638.

17 Cannon R, Ellis S, Hayes D, Narayanan G, Martin RC 2nd: Safety and early efficacy of irreversible electroporation for hepatic tumors in proximity to vital structures. J Surg Oncol 2013;107:544-549.

18 Silk MT, Wimmer T, Lee KS, et al: Percutaneous ablation of peribiliary tumors with irreversible electroporation. J Vasc Interv Radiol 2014;25:112-118.

19 Scheffer HJ, Nielsen K, de Jong MC, et al: Irreversible electroporation for nonthermal tumor ablation in the clinical setting: a systematic review of safety and efficacy. J Vasc Interv Radiol 2014;25:997-1011. 